\title{
Gliquidone improves retinal injury to relieve diabetic retinopathy via regulation of SIRT1/ Notch1 pathway
}

\author{
Mengdan Yu, Lijun Zhang, Shasha Sun and Zhenhua Zhang*
}

\begin{abstract}
Background: Diabetic retinopathy (DR) is a common and potentially devastating microvascular complication of diabetes mellitus (DM). The main features of DR are inflammation and oxidative damage. Gliquidone (GLI) is confirmed to be a hypoglycemic drug by oral administration. The current study is aimed to investigate the role and mechanism of GLI on the pathogenesis of DR.

Methods: High glucose (HG)-induced human retinal endothelial cells (HRECs) were used to explore the anti-inflammatory and anti-oxidant effects of GLI on DR in vitro. Streptozotocin (STZ)-induced DM rats were used to investigate the effects of GLI on retinal structures, inflammation, and oxidative stress. The levels of SIRT1/Notch1 pathway-related proteins were determined by western blotting.

Results: GLI treatment promoted the viability and inhibited the apoptosis of HG-induced HRECs. Meanwhile, the levels of interleukin (IL)-6, IL-1 $\beta$, tumour necrosis factor alpha and reactive oxygen species were suppressed, while both catalase and superoxide dismutase were elevated after GLI treatment in HG-induced HRECs. Furthermore, we found that Silencing information regulator 2 related enzyme 1 (SIRT1) silencing reversed the inhibiting effects of GLI on the levels of protein Notch1 and effector genes Hes 1 and Hey2. Similar anti-inflammatory and anti-oxidant effects of GLI in STZ-induced DM rats were observed. Additionally, GLI administration also repressed vascular hyperpermeability in vivo.
\end{abstract}

Conclusion: GLI may be an effective agent to improve DR through repression of inflammation and oxidative stress via SIRT1/Notch1 pathway.

Keywords: Gliquidone, diabetic retinopathy, inflammation, oxidative stress, SIRT1/Notch1 pathway

\section{Background}

Diabetic retinopathy (DR) is a severe microvascular complication for diabetes mellitus (DM) patients, which is a leading cause of vision impairment for working-age adults worldwide [1, 2]. High level of blood glucose is the main contributing factor to affect DR progression, triggering pathological changes of the retinal cells [3]. In the

${ }^{*}$ Correspondence: Shuijing20212021@126.com

Department of Ophthalmology, Affiliated Qingdao Central Hospital, Qingdao University, No. 127, Siliu South Road, Qingdao City 266042,

Shandong Province, China pathogenesis of DR, endothelial dysfunction, inflammation, and oxidative stress are the three main pathological features [4-6]. Currently, the prevalent treatments for retinal vascular dysfunction such as intraocular injection of anti-vascular endothelial growth factor (VEGF) drugs and steroids have developed rapidly [7-10]. For example, intraocular glucocorticoids are approved for clinical use to treat diabetic macular edema, and topical ocular treatment has been explored with novel selective glucocorticoid receptor agonists [7]. But some adverse effects including retinal neuron destruction, irreversible retinal damage, and even vision loss are still occurred [8-10]. original author(s) and the source, provide a link to the Creative Commons licence, and indicate if changes were made. The images or other third party material in this article are included in the article's Creative Commons licence, unless indicated otherwise in a credit line to the material. If material is not included in the article's Creative Commons licence and your intended use is not permitted by statutory regulation or exceeds the permitted use, you will need to obtain permission directly from the copyright holder. To view a copy of this licence, visit http://creativecommons.org/licenses/by/4.0/. The Creative Commons Public Domain Dedication waiver (http://creativeco mmons.org/publicdomain/zero/1.0/) applies to the data made available in this article, unless otherwise stated in a credit line to the data. 
Gliquidone (GLI) is an anti-diabetic medication approved for the clinical use and it is classified as a second-generation sulfonylurea [11-13]. However, researches on the therapeutic efficacy of GLI on DR are relatively rare.

Silent information regulator 2 (SIR2), belonging to the histone deacetylases family, functions as anti-inflammation and oxidation resistance [14]. There are seven homologues of SIR2 named as SIRT1, SIRT2, SIRT3, SIRT4, SIRT5, SIRT6, and SIRT7, respectively. SIRT1 has been confirmed to be deeply involved in the progression of DR $[15,16]$. For instance, Jiang et al. found that resveratrol, a specific activator of SIRT1, can remarkably promote the viability of human retinal endothelial cells (HRECs) and repress the secretion of inflammatory cytokines [15]. Ji et al. conducted a DR rat model and demonstrated a decreased expression of SIRT1 in high glucose-induced rat retinal endothelial cells (RRECs) and retinal tissues of DR rats [16]. Meanwhile, they further indicated that overexpressed SIRT1 can accelerate the proliferation of RRECs [16]. Additionally, Notch1 signaling is reported as a downstream effector pathway of SIRT1 to affect the development of human diseases [17-19]. Notch signaling family is a well-known conserved pathway to regulate inflammatory reactions and oxidative stress, [20] and cellular processes such as proliferation, differentiation, and apoptosis [21]. Meanwhile, Dou et al. believed that Notch signaling plays vital roles in retinal angiogenic sprouting/vasculature maturation/vascular stability [22]. As one of the important receptors of Notch, the activation of Notch1 is confirmed to induce vascular permeability, which increases the risk of DR [23]. More importantly, a latest research revealed that SIRT1 can inactivate Notch1 signaling, eventually alleviating the development of sepsis [24]. Tian et al. constructed a diabetic nephropathy (DN) mouse model and showed that GLI may improve oxidative damage and renal interstitial fibrosis to delay the progression of DN through inhibiting Notch1 signaling [25]. However, whether GLI interacts with SIRT1/ Notch1 pathway to affect DR progression is still unclear.

In this study, the function of GLI and its relationship with SIRT1/Notch1 pathway in the progression of DR were preliminarily investigated. These results may provide a clinical therapeutic method for DR.

\section{Methods}

\section{Reagents}

Angio-proteomie (Boston, MA, USA) provided human retinal endothelial cells (HRECs). Cell culture and transfection-related reagents were from Invitrogen (Carlsbad, CA, USA). SIRT1-siRNA (si-SIRT1-1/-2) and the negative control (si-NC) were from Sangon Biotech (Shanghai, China). The commercial kits for the measurement of glucose and triglyceride contents were from
Boxbio (Beijing, China). The caspase-3 assay kit was from QCbio Science \& Technologies (Shanghai, China). Sigma Aldrich (San Luis, MO, USA) provided streptozotocin (STZ) and the commercial kits for the measurement of reactive oxygen species (ROS), catalase (CAT), superoxide dismutase (SOD), and inflammatory cytokines. 3-(4, 5-dimethyl-2-thiazolyl)-2, 5-diphenyl-2-h-tetrazolium bromide (MTT) was from Aladdin (Shanghai, China). Apoptosis detection kit and western blotting analysisrelated reagents were from Thermo Fisher Scientific (Waltham, MA, USA). Rat VEGF ELISA kit, the primary antibodies (Notch1, Hes1, Hey2, SIRT1, and GAPDH), and the HRP-conjugated secondary antibody were procured from Abcam (Cambridge, UK).

\section{DM in vitro model}

HRECs were cultured in Dulbecco's modified Eagle's medium containing $10 \%$ fatal bovine serum, $1 \%$ penicillin/streptomycin at $37^{\circ} \mathrm{C}$ with $5 \% \mathrm{CO}_{2}$. Afterwards, HRECs were incubated with normal glucose (NG; 5 $\mathrm{mmol} / \mathrm{L}$ ), high glucose (HG; $30 \mathrm{mmol} / \mathrm{L}$ ), and HG (30 $\mathrm{mmol} / \mathrm{L})+$ GLI $(1 \mu \mathrm{g} / \mathrm{ml})$, respectively. Lipofectamine 3000 was used to perform transfection experiments.

\section{Measurement for the contents of glucose and triglyceride, and glucose uptake}

According to the manufacturer's instructions, the glucose and triglyceride contents in HRECs of each group were measured using the corresponding commercial kits. The results were assessed using a spectrophotometer at 505 $\mathrm{nm}$ (glucose) and $420 \mathrm{~nm}$ (triglyceride), respectively. In addition, the 2-deoxy-D-glucose-6-phosphate (2DG6P) uptake assay was performed in strict accordance with the previous study [26].

\section{Cell viability assay}

HRECs (10000 per well) were seeded in 96-well plates for $24 \mathrm{~h}$ at $37^{\circ} \mathrm{C}, 5 \% \mathrm{CO}_{2}$, followed by adding MTT $(5 \mathrm{mg} /$ $\mathrm{ml}$ ) to each well. After incubation for $4 \mathrm{~h}$, the absorbance at $450 \mathrm{~nm}$ was measured.

\section{Caspase- 3 activity assay}

The activity of caspase-3 in HRECs was assessed using a caspase-3 assay kit under a micro-plate reader with the absorbance of $405 \mathrm{~nm}$.

\section{Cell apoptosis assay}

Cell apoptosis was assessed by flow cytometric analysis. In brief, cells $\left(1 \times 10^{5}\right.$ cells $\left./ \mathrm{mL}\right)$ were cultured in 96-well plates for $24 \mathrm{~h}$, and then stained with V-FITC and PI using an apoptosis detection kit at $25^{\circ} \mathrm{C}$ for $20 \mathrm{~min}$ in the dark. The apoptotic cells were measured using a flow cytometer (BD Biosciences). 


\section{Animal model}

All animal experiments in this study were in strict accordance with the protocols stated in the Guide for the Care and Use of Laboratory Animals and approved by the ethical committee of Affiliated Qingdao Central Hospital, Qingdao University. A total of 24 male Sprague Dawley (SD) rats (200-220 g, 10 weeks) purchased from EseBio (Shanghai, China) was allowed to adapt to the laboratory environment before testing. One week later, the rats were assigned into three groups $(n=8)$ : the normal group, the DM group, and the DM + GLI group. STZ (60 mg/kg body weight) dissolved in citrate buffer (10 mM) was intraperitoneally (i.p.) injected into rats to induce DM, while the rats injected with equal volume of citrate buffer were served as the normal group. The level of fasting blood glucose that more than $16.7 \mathrm{mmol} / \mathrm{L}$ was served as the DM rats. Afterwards, GLI $(50 \mathrm{mg} / \mathrm{kg}$ body weight per day) dissolved in dimethylformamide (DMF) was administrated to rats in the DM + GLI group lasting for 12 weeks by intragastric administration [13]. The rats in the normal and DM groups were administrated with equal volume of DMF.

\section{Assessment for retinal vascular permeability}

After GLI administration, rats in each group were anesthetized by pentobarbital sodium ( $200 \mathrm{mg} / \mathrm{kg}$; i.p.) and then administered with Evans blue dye (30 mg/ $\mathrm{kg})$ via femoral vein injection. Subsequently, phosphate buffer saline was perfused into the left ventricle of rats and then $4 \%$ paraformaldehyde. The retinal tissues were then collected. Evans blue dye was extracted in formamide and quantified by absorbance measurement at $420 \mathrm{~nm}$.

\section{Analysis for inflammation, oxidative stress and VEGF level} According to the manufacturer's instructions, the levels of interleukin (IL)-6, IL-1 $\beta$, tumour necrosis factor alpha (TNF-) $\alpha$, ROS, CAT, SOD, and VEGF in retina tissues of DM rats and/or HG-induced HRECs were measured by the corresponding commercial kits.

\section{VEGF mRNA level measurement}

The mRNA level of VEGF was detected by quantitative real time PCR (qRT-PCR). In brief, total RNA extracted from retinal tissues of DM rats was used for cDNA synthesis and then for qRT-PCR analysis. The $2^{-\Delta \Delta C t}$ method was utilized to calculate the VEGF mRNA level and GAPDH was used as the internal control.

\section{Hematoxylin-eosin (HE) staining}

The retinal tissues were fixed in $4 \%$ paraformaldehyde for $24 \mathrm{~h}$, followed by embedding in paraffin sectioned at
$5 \mu \mathrm{m}$ thickness. All the sections were stained with $\mathrm{H} \& \mathrm{E}$ staining immediately and then were observed by a light microscopy.

\section{Western blot}

Proteins were extracted from HG-induced HRECs and retinal tissues of DM rats using RIPA lysis buffer. The protein concentrations were then determined using a BCA kit. Then proteins were analyzed by $10 \%$ polyacrylamide gel electrophoresis, and transferred to PVDF membranes. Before the primary antibodies incubation, the membrane was blocked using 5\% nonfat milk. Then the membrane was incubated with the secondary antibody for $1 \mathrm{~h}$, and analyzed by ECL kit. GAPDH was internal reference of Notch1, Hes1, Hey2, and SIRT1.

\section{Statistical analysis}

The data were analyzed using SPSS 22.0 software and expressed in the form of mean \pm SD. Student's t-test and one-way ANOVA followed by Tukey's multiple comparisons test were used for data analysis. $P$ values $<0.05$ were considered statistically significant.

\section{Results}

HG treatment increases the contents of glucose, triglyceride, and 2DG6P in HRECs

The contents of glucose, triglyceride, and 2DG6P in HGinduced HRECs were measured. As illustrated in Fig. 1A$C$, we found that compared to the NG groups, HG treatment significantly increased the contents of glucose, triglyceride, and 2DG6P in HRECs $(P<0.01)$, suggesting that a DM cell model was successfully established.

\section{GLI promotes cell viability but inhibits the apoptosis of HG-induced HRECs}

The influences of GLI on HREC viability and apoptosis under HG conditions were then explored. We demonstrated that in HRECs, HG treatment remarkably decreased the cell viability, but promoted caspase- 3 activity and apoptosis (Fig. $2 \mathrm{~A}-\mathrm{C}, P<0.01$ ). After addition of GLI, HG-induced HRECs showed the increased cell viability and the reduced caspase- 3 activity and apoptosis rate $(P<0.01)$.

\section{GLI suppresses the inflammatory responses and oxidative stress caused by HG treatment in HRECs}

Both inflammation and oxidative damage are confirmed to affect DR progression $[27,28]$. Therefore, the effects of GLI on inflammatory cytokines (IL-6, IL-1 $\beta$, and TNF$\alpha)$ and oxidative stress-related factors (ROS, CAT, and SOD) in HG-induced HRECs were further investigated. As expected, HG treatment significantly elevated the levels of IL- 6 , IL-1 $\beta$, TNF- $\alpha$ and ROS, and repressed the 


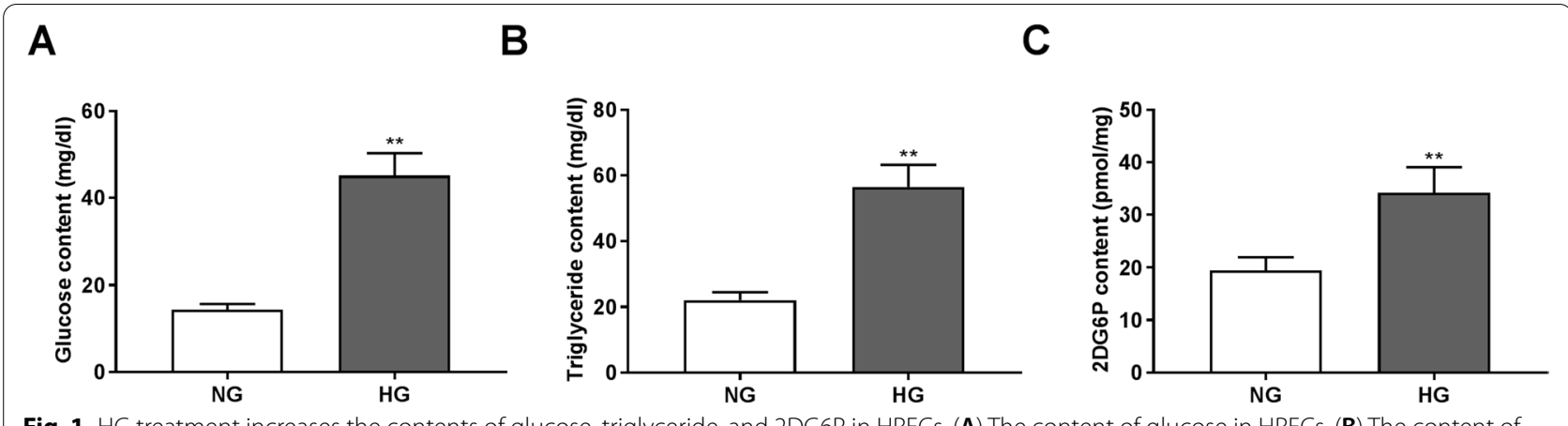

Fig. $1 \mathrm{HG}$ treatment increases the contents of glucose, triglyceride, and 2DG6P in HRECs. (A) The content of glucose in HRECs. (B) The content of triglyceride in HRECs. (C) The glucose uptake in HRECs. ${ }^{* *} P<0.01$ vs. the NG group. $\mathrm{HG}: 30 \mathrm{mmol} / \mathrm{L}$ glucose. NG: $5 \mathrm{mmol} / \mathrm{L}$ glucose

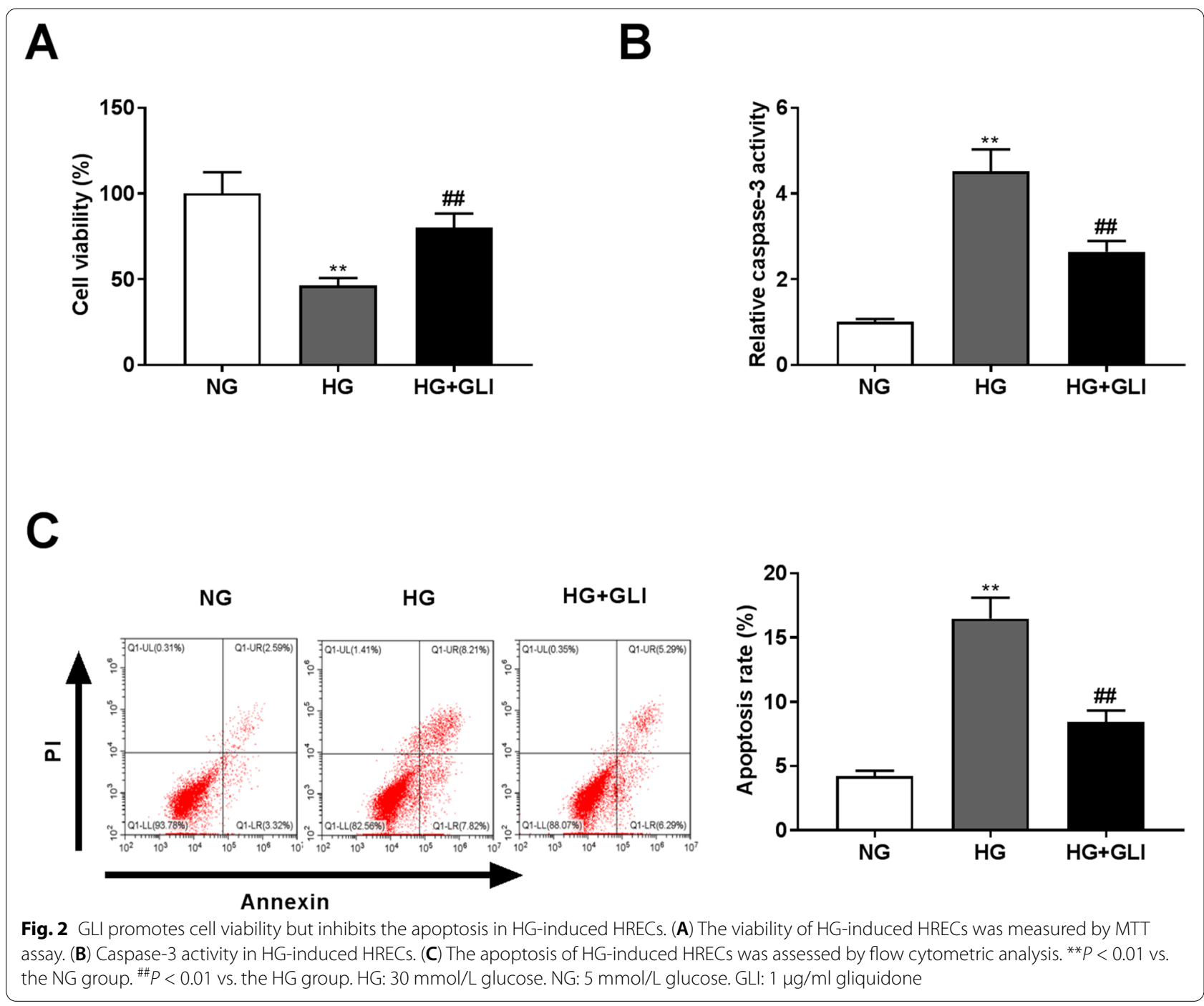

release of CAT and SOD (Fig. 3A-F, $P<0.01$ ). However, these situations were all reversed by GLI treatment $(P<$ 0.05).
GLI represses Notch1 signaling in HG-induced HRECs

Numerous studies have reported that Notch1 signaling is deeply involved in the pathogenesis of DR via regulation 


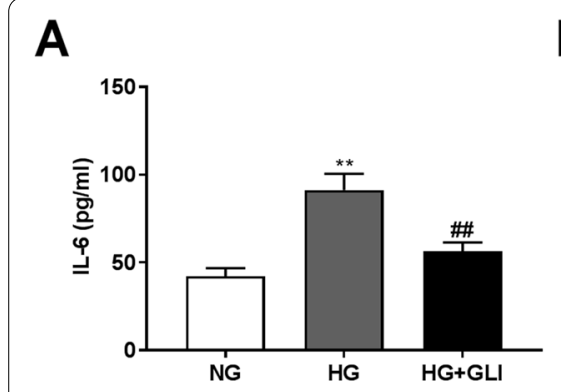

B

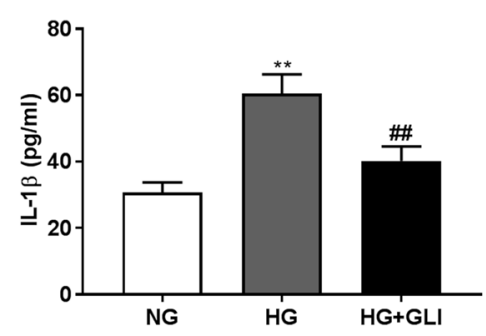

E

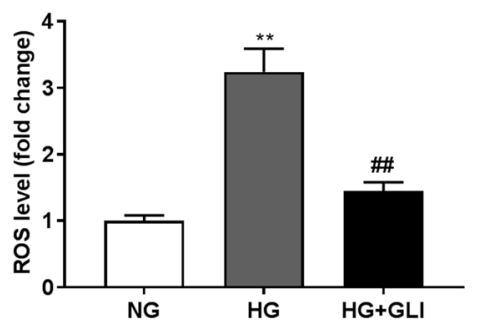

C

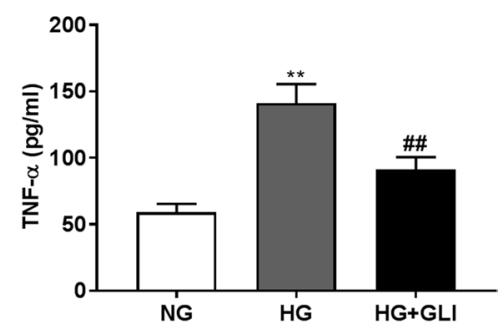

$\mathbf{F}$

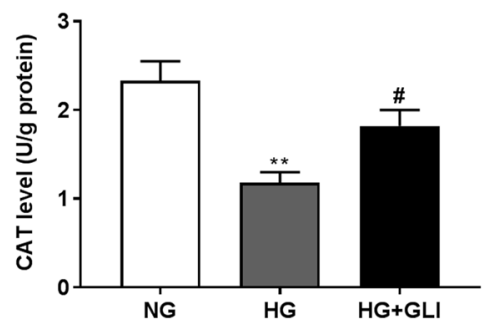

Fig. 3 GLI suppresses the inflammatory responses and oxidative stress caused by HG in HRECs. (A) The level of IL-6 in HG-induced HRECs was measured by ELISA assay. (B) The level of IL- $\beta$ in HG-induced HRECs was measured by ELISA assay. (C) The level of TNF-a in HG-induced HRECs was measured by ELISA assay. (D) The level of ROD in HG-induced HRECs was measured by a commercial kit. (E) The level of CAT in HG-induced HRECs was measured by a commercial kit. (F) The level of SOD in HG-induced HRECs was measured by a commercial kit. ${ }^{* *} P<0.01$ vs. the NG group. ${ }^{\#} P<$ 0.05, ${ }^{\# \# P<0.01 ~ v s . ~ t h e ~ H G ~ g r o u p . ~ H G: ~} 30$ mmol/L glucose. NG: 5 mmol/L glucose. GLI: 1 $\mu \mathrm{g} / \mathrm{ml}$ gliquidone

of inflammation and vascular permeability [23, 29]. The interactions between GLI and Notch1 signaling-related genes were then studied. Western blotting analysis showed that the protein levels of Notch1, Hes1 and Hey2 were significantly increased in the $\mathrm{HG}$ group relative to the NG group (Fig. 4, $P<0.01$ ). Addition of GLI reversed the promoting effects of HG treatment on the levels of these proteins $(P<0.01)$.

\section{GLI interacts with Notch1/SIRT1 pathway in HG-induced HRECS}

The inhibiting effect of SIRT1 on Notch-mediated transcription has been confirmed in renal injured endothelial cells [30]. As presented in Fig. 5A, we indicated that the protein level of SIRT1 was remarkably downregulated in the HG group compared to that in NG group $(P<0.01)$. Meanwhile, GLI eliminated the suppressive effect of HG on SIRT1 protein level $(P<$ 0.01). Then, si-SIRT1-1/-2 was transfected into HRECs to determine the transfection efficiency. Western blotting revealed that SIRT1 protein level was significantly reduced in HRECs after transfection (Fig. 5B, $P<0.01$ ). We chose si-SIRT1-1 for the subsequent experiments due to the relatively high transfection efficiency. As expected, transfection of si-SIRT1-1 partly eliminated the inhibitory effects of GLI on the levels of Notch1,
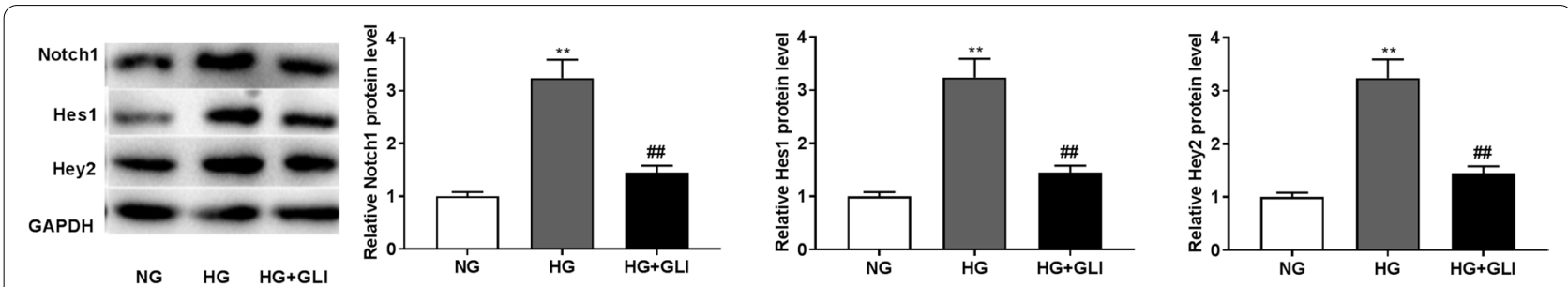

Fig. 4 GLI inactivates Notch1 signaling in HG-induced HRECs. The protein levels of Notch1, Hes1, and Hey2 in HG-induced HRECs. ${ }^{*} P<0.01$ Vs. the NG group. ${ }^{\#} P$ < 0.01 vs. the HG group. HG: 30 mmol/L glucose. NG: 5 mmol/L glucose. GLI: 1 mg/ml gliquidone 

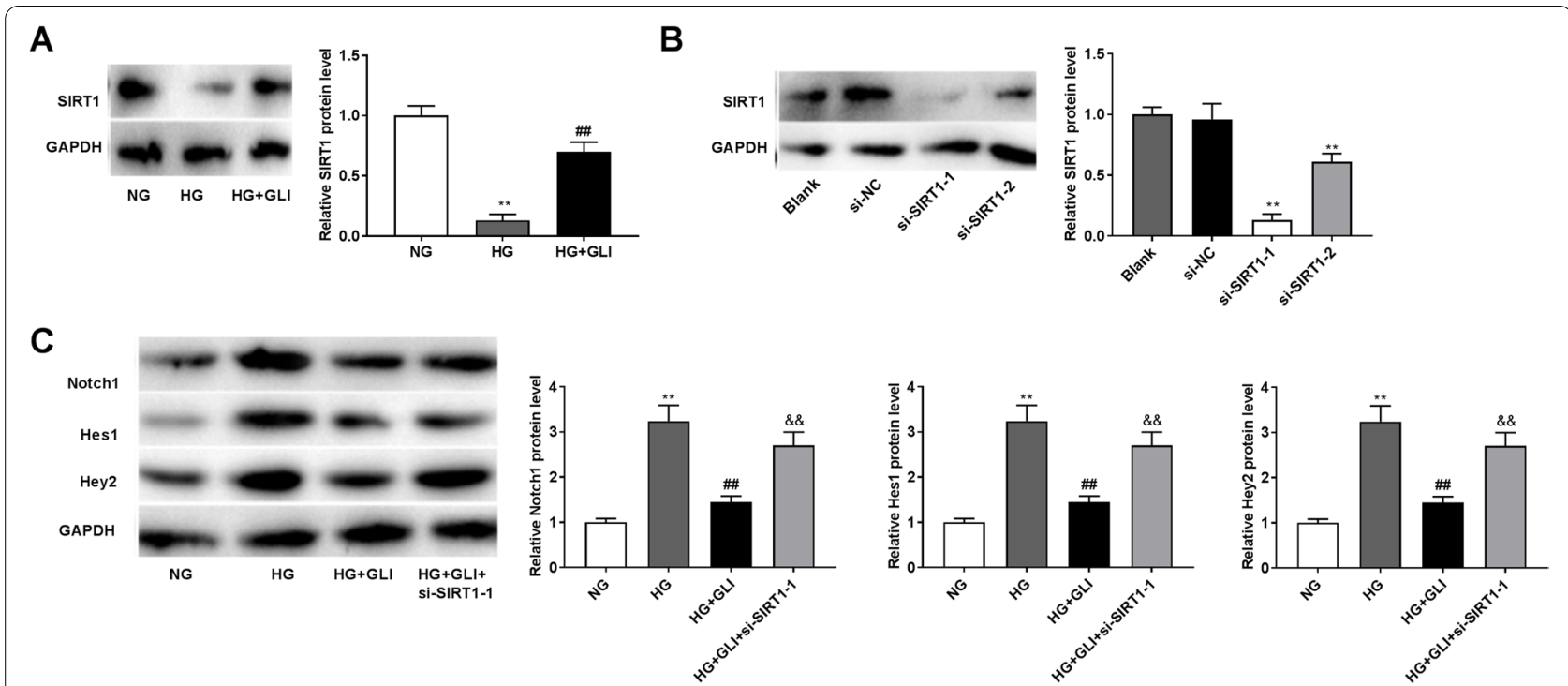

Fig. 5 GLI interacts with Notch1/SIRT1 pathway in HG-induced HRECs. (A) The protein level of SIRT1 in HG-induced HRECs was determined by western blotting. ${ }^{* *} P<0.01$ vs. the NG group. ${ }^{\# \#} P<0.01$ vs. the HG group. (B) The protein level of SIRT1 after transfection of si-SIRT1-1/-2/NC was determined by western blotting. ${ }^{* *} P<0.01$ vs. the si-NC group. (C) The protein levels of Notch1, Hes 1, and Hey2 in HG-induced HRECs transfected with si-SIRT1-1. ${ }^{* *} P<0.01$ vs. the NG group. ${ }^{\# \#} P<0.01$ vs. the $\mathrm{HG}$ group. ${ }^{\&} \&>0.01$ vs. the $\mathrm{HG}+\mathrm{GLI}$ group. HG: $30 \mathrm{mmol} / \mathrm{L}$ glucose. NG: $5 \mathrm{mmol} / \mathrm{L}$ glucose. GLI: $1 \mu \mathrm{g} / \mathrm{ml}$ gliquidone

Hes1, and Hey2 in HG-induced HRECs (Fig. 5C, $P<$ $0.01)$.

\section{GLI administration attenuates retinal injury in STZ-induced DM rats}

A DM rat model was established to further explore the therapeutic effect of GLI on DR in vivo. The normal group showed the intact retinal structure. A decrease in retinal thickness was found in rats of the DM group (Fig. 6A, $P<0.01$ ). However, GLI administration improved the morphological changes in the retinas $(P<$ $0.05)$. The increased vascular permeability can result in retinopathy and vision loss [31]. The Evans blue staining assay was used to assess the retinal vascular permeability in STZ-induced DM rats. We found a significant increase in the retinal vascular permeability of DM rats (Fig. 6B, $P<0.01)$. As expected, after administration of GLI, the retinal vascular permeability was sharply decreased $(P$ $<0.01$ ). As previously described, VEGF plays important role in retinal vascular permeability [32]. Therefore, the level of VEGF was then detected. As shown in Fig. 6C$\mathrm{D}$, both the mRNA expression and protein level of VEGF were elevated in the DM group $(P<0.01)$ but were reduced in the DM + GLI group $(P<0.01)$.

\section{GLI inhibits inflammation and oxidative injury in retinal tissues of DM rats via SIRT1/Notch1 pathway}

The effects of GLI administration on inflammation and oxidative injury in retinal tissues of DM rats were also explored. Similar to the results of in vitro model, DM rats showed relatively high levels of IL-1 $\beta$, TNF- $\alpha$, IL- 6 and ROS, and decreased levels of CAT and SOD (Fig. 7AF, $P<0.01)$. GLI administration partly improved these adverse impacts $(P<0.01)$. In addition, we also found that compared to the normal group, rats in the DM group had decreased protein level of SIRT1, and increased Notch1, Hes1 and Hey2 protein levels (Fig. 7G, $P<0.01$ ). GLI administration also reversed the effects of DM on the levels of these proteins $(P<0.01)$.

\section{Discussion}

More than $80 \%$ DM patients may suffer from DR at the first two decades of diabetes [34]. Although the management opinions for DR have greatly improved, some severe side effects are still confirmed to affect the therapeutic efficacy [8-10]. Exploring effective drugs to furthest decrease the side effects in the treatment of DR is extremely urgent. GLI is a well-known hypoglycemic agent in clinic [11]. Previous study has revealed the therapeutic effect of GLI on diabetic nephropathy [25]. But there still no researches on the possible function of GLI on DR progression. This study for the first time uncovers that GLI can relieve DR through regulation of endothelial dysfunction, inflammation, and oxidative stress via SIRT1/Notch1 pathway, suggesting that GLI may be an effective agent for the treatment of DR in clinic.

HG-induced HRECs are generally used as an in vitro model of DR due to the crucial role in angiogenesis 


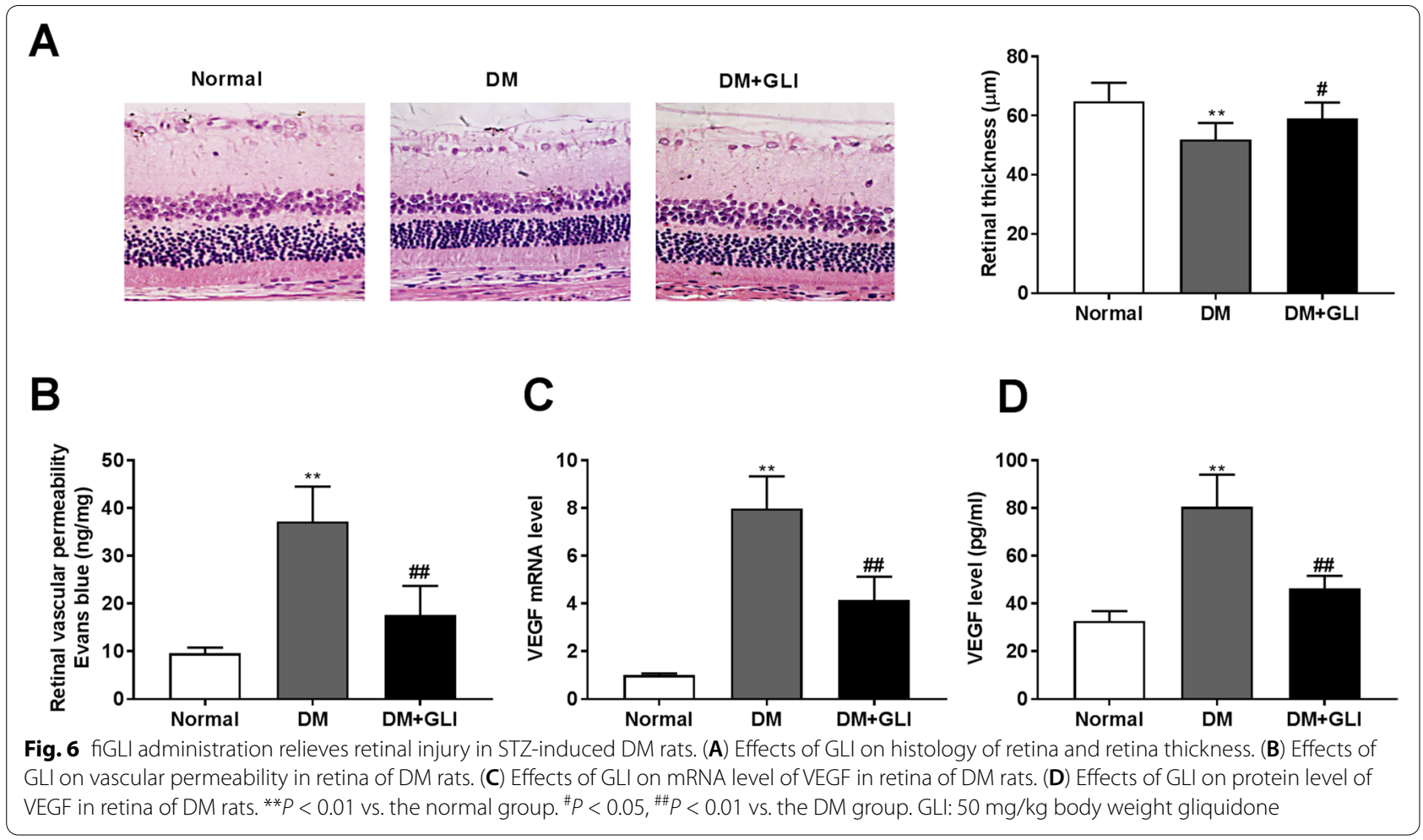

[29, 35, 36]. In this study, HG treatment significantly increased the contents of glucose, triglyceride and 2DG6P in HRECs, which is consistent with the data of Long et al. [36]. The results suggested that DR cell model was established successfully. The pathological feature of DR is not only manifested as the increased level of blood glucose, but also the selective apoptosis of endothelial cells [37]. Similarly, we demonstrated that in HG-induced HRECs, the cell viability was decreased, while the apoptosis was promoted. Addition of GLI promoted cell viability and repressed apoptosis of HG-induced HRECs. The experiments indicated that GLI treatment can effectively inhibit the development of DR in a cellular level. Meanwhile, our in vivo experiments that GLI administration improved the decreased retinal thickness caused by DM. Interestingly, Zhu et al. investigated the effect of TGR5 receptor agonist (INT-777) on morphology of retinal tissues in a STZ-induced rat model and found a decrease in retinal thickness induced by diabetes, while intravitreal injection of INT-777 alleviated such morphological changes in the retinas, suggesting that TGR5 agonism is protective against diabetes-induced retinal vascular injury [38]. As a result, we also believed that GLI can alleviate retinal vascular injury in the progression of DR. As well accepted, excessive inflammation responses and oxidative damage are exited in the progression of DR $[27,28]$. In the current study, we found enhanced inflammatory reactions and oxidative injuries in HGinduced HRECs and STZ-induced DM rats. In addition, we also demonstrated inflammatory reactions and oxidative stress were relieved by GLI administration both in vitro and in vivo. Similarly, $\mathrm{Li}$ et al. focused on the protective effects of sulforaphane on inflammation and peroxidation both in vitro and in vivo, and demonstrated that sulforaphane can attenuate the development of DR through inhibiting inflammation and oxidative stress in HG-induced rat Müller cells and STZ-induced DM rats [39]. Combined the previous results, we concluded that GLI may be also an effective agent to repress inflammation and oxidative stress in DR progression. Vascular hyperpermeability is the initial pathologic feature in DR capillaries, [40] and the increased level of VEGF is confirmed to be closely associated with retinal vascular permeability [32]. Previous researches have confirmed that some agents such as blueberry anthocyanins [41], resveratrol [42], and melatonin [43] can modulate vascular permeability via suppression of VEGF level in DR rats. In this investigation, as expected, DM rats showed relatively high level of VEGF, while administration of GLI repressed the release of VEGF. We speculated that GLI may be helpful for the inhibition of vascular hyperpermeability in the progression of DR. Based on the above data, we believed that GLI may be also an effective agent to ameliorate DR through suppressing a series of 


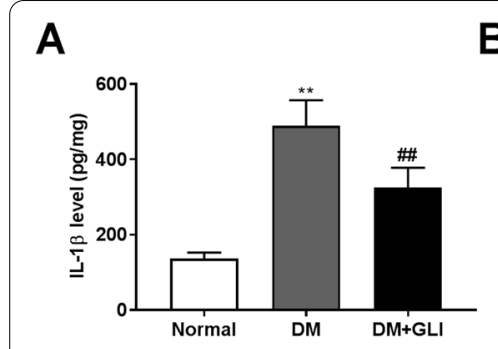

B

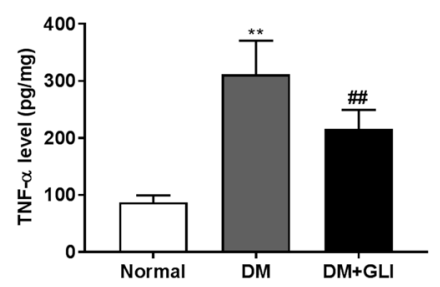

E
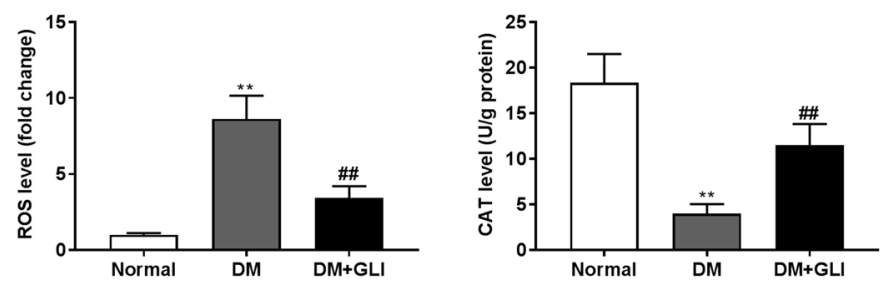

C

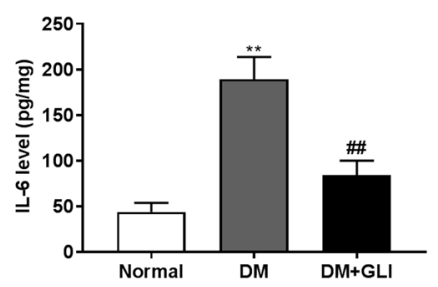

$\mathbf{F}$

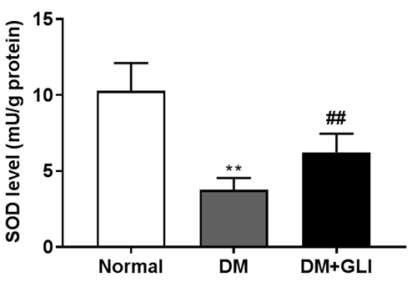

G
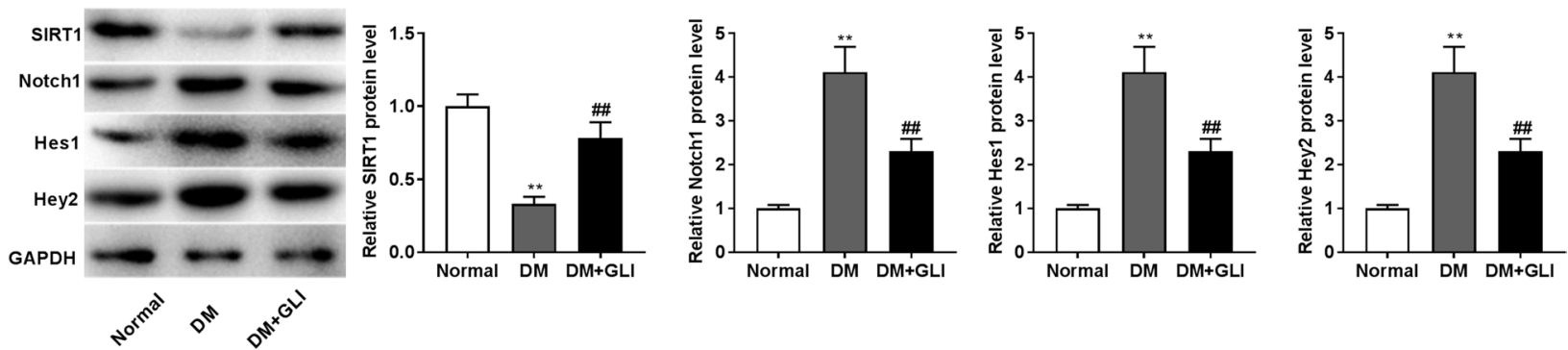

Fig. $7 \mathrm{GLI}$ represses inflammation and oxidative injury in retinal tissues of DM rats via SIRT1/Notch1 pathway. (A) The level of IL- $\beta$ in retina of DM rat after GLI administration was measured by ELISA assay. (B) The level of TNF-a in retina of DM rat after GLI administration was measured by ELISA assay. (C) The level of IL-6 in retina of DM rat after GLI administration was measured by ELISA assay. (D) The level of ROS in retina of DM rat after GLI administration was measured by a commercial kit. (E) The level of CAT in retina of DM rat after GLI administration was measured by a commercial kit. (F) The level of SOD in retina of DM rat after GLI administration was measured by a commercial kit. (G) The protein levels of SIRT1, Notch1, Hes 1, and Hey2 in retina of DM rat after GLI administration was determined by western blotting. ${ }^{*} P<0.01$ vs. the normal group. ${ }^{\# \#} P<0.01$ vs. the DM group. GLI: $50 \mathrm{mg} / \mathrm{kg}$ body weight gliquidone

pathological processes of DR including endothelial cell apoptosis, inflammation, oxidative damage, and vascular hyperpermeability. In clinic, GLI may inhibit endothelial dysfunction, inflammation responses, oxidative injuries and vascular hyperpermeability, thereby contributing to the improvement of poor prognosis of DR patients.

Notch1 signaling and the corresponding effector genes act as important roles in prevention of inflammation and oxidative stress in numerous human diseases, such as arthritis, [44] enteritis, [45] myocardial injury, [46] and chronic obstructive pulmonary disease [47]. We speculated that there may be some relationships between GLI and Notch1 signaling. As expected, the in vitro and in vivo experiments showed that the protein levels of Notch1 and effector genes (Hes1 and Hey2) were elevated in HG-induced HRECs and STZ-induced DM rats, which suggested that the activation Notch1 signaling may accelerate the development of DR. Similarly, Miloudi et al. believed that the activation of Notch1 increases the risk of DR via inducing vascular permeability [23]. Additionally, we further indicated that GLI partly eliminated the promoting effects of HG on Notch1 signaling-related proteins. The results implied that GLI can repress the activation of Notch1 signaling. Notch1 signaling has been reported as a downstream effector pathway of SIRT1 to affect disorder progression, such as liver fibrosis, breast cancer, and lung cancer [17-19]. We then further speculated GLI may also interact with SIRT1 in DR progression. Firstly, we found that SIRT1 protein level was downregulated in HG-induced HRECs and DM 
rats. Similarly, a decreased expression of SIRT1 is found in HG-induced RRECs and retinal tissues of DR rats [16]. However, GLI treatment significantly elevated the protein level of SIRT1, suggesting that GLI may induce the production of SIRT1. Based on the results that GLI can inactivate Notch1 signaling, we suggested that GLI may interact with SIRT1/Notch1 pathway to affect DR progression. As expected, we found that downregulation of SIRT1 partly attenuated the inhibiting effects of GLI on the levels of Notch1 signaling-related proteins. Therefore, the SIRT1/Notch1 signaling may be an important regulatory pathway in DR, providing a direction for the research of novel targeted drugs in clinic. Assuredly, except for SIRT1/Notch1 pathway, there are numerous signaling pathways involved in DR progression, such as PIGF/ERK [48], PLA2/COX-2/VEGF-A [49], TLR4/NFkappaB [50], and PI3K/Akt/mTOR [51]. We speculated GLI may also ameliorate DR via these pathways. This is may be a limitation of this study and we will elucidate these issues in future studies. Additionally, some other factors have been linked to the progression of DR such as miRNAs $[52,53]$ and lncRNAs $[54,55]$. The interaction of GLI with them should be further explored.

\section{Conclusions}

In summary, the present investigation indicates that GLI may be an effective agent to improve DR through inhibiting inflammation and oxidative stress via SIRT1/Notch1 pathway. Our findings clarify the action mechanism of GLI on the development of DR, and may provide an effective drug for DR therapy in clinic.

\section{Abbreviations}

DR: Diabetic retinopathy; DM: diabetes mellitus; GLI: Gliquidone; HG: High glucose; HRECs: human retinal endothelial cells; STZ: Streptozotocin.

\section{Acknowledgements}

Not applicable.

\section{Consent to publish}

Not applicable.

\section{Authors' contributions}

MDY designed and analyzed the experiment, performed the experiment, wrote the manuscript and approved the final manuscript. LJZ: performed the experiment, revised the manuscript and approved the final manuscript. SSS: performed the experiment, wrote the manuscript and approved the final manuscript. ZHZ: performed the experiment, revised the manuscript and approved the final manuscript. The authors read and approved the final manuscript.

\section{Funding}

Not applicable.

\section{Availability of data and materials}

The datasets used and/or analysed during the current study are available from the corresponding author on reasonable request.

\section{Declarations}

Ethics approval and consent to participate

This study was conducted after obtaining Affiliated Qingdao Central Hospital, Qingdao University's ethical committee approval.

The study is reported in accordance with ARRIVE guidelines

\section{Competing interests}

The authors declare that they have no competing interests.

Received: 3 September 2021 Accepted: 7 December 2021

Published online: 27 December 2021

\section{References}

1. Song SJ, Han K, Choi KS, Ko SH, Rhee EJ, Park CY, et al. Trends in diabetic retinopathy and related medical practices among type 2 diabetes patients: Results from the National Insurance Service Survey 2006-2013. J Diabetes Investig. 2018;9(1):173-8. https://doi.org/10.1111/jdi.12655.

2. Fan Y, Qiao Y, Huang J, Tang M. Protective Effects of Panax notoginseng Saponins against High Glucose-Induced Oxidative Injury in Rat Retinal Capillary Endothelial Cells. Evid Based Complement Alternat Med. 2016:2016:5326382. https://doi.org/10.1155/2016/5326382.

3. Wang CF, Yuan JR, Qin D, Gu JF, Zhao BJ, Zhang L, et al. Protection of tauroursodeoxycholic acid on high glucose-induced human retinal microvascular endothelial cells dysfunction and streptozotocin-induced diabetic retinopathy rats. J Ethnopharmacol. 2016;185:162-70. https:// doi.org/10.1016/j.jep.2016.03.026.

4. Lu JM, Zhang ZZ, Ma X, Fang SF, Qin XH. Repression of microRNA-21 inhibits retinal vascular endothelial cell growth and angiogenesis via PTEN dependent-PI3K/Akt/NEGF signaling pathway in diabetic retinopathy. Exp Eye Res. 2020;190:107886. https://doi.org/10.1016/j.exer.2019. 107886.

5. Rubsam A, Parikh S, Fort PE. Role of Inflammation in Diabetic Retinopathy Int J Mol Sci. 2018;19(4). https://doi.org/10.3390/ijms1 9040942.

6. Calderon GD, Juarez OH, Hernandez GE, Punzo SM, De la Cruz ZD. Oxidative stress and diabetic retinopathy: development and treatment. Eye (Lond). 2017:31(8):1122-30. https://doi.org/10.1038/eye.2017.64.

7. Shafiee A, Bucolo C, Budzynski E, Ward KW, Lopez FJ. In vivo ocular efficacy profile of mapracorat, a novel selective glucocorticoid receptor agonist, in rabbit models of ocular disease. Invest Ophthalmol Vis Sci. 2011;52(3):1422-30. https://doi.org/10.1167/iovs.10-5598.

8. Royle P, Mistry H, Auguste P, Shyangdan D, Freeman K, Lois N, et al. Pan-retinal photocoagulation and other forms of laser treatment and drug therapies for non-proliferative diabetic retinopathy: systematic review and economic evaluation. Health Technol Assess. 2015;19 (51):v-xxviii:1-247. https://doi.org/10.3310/hta19510.

9. He F, Xia X, Wu XF, Yu XQ, Huang FX. Diabetic retinopathy in predicting diabetic nephropathy in patients with type 2 diabetes and renal disease: a meta-analysis. Diabetologia. 2013;56(3):457-66. https://doi.org/10.1007/ s00125-012-2796-6.

10. Banji D, Pinnapureddy J, Banji OJ, Saidulu A, Hayath MS. Synergistic activity of curcumin with methotrexate in ameliorating Freund's Complete Adjuvant induced arthritis with reduced hepatotoxicity in experimental animals. Eur J Pharmacol. 2011;668(1-2):293-8. https://doi.org/10.1016/j. ejphar.2011.06.006.

11. Malaisse WJ. Gliquidone contributes to improvement of type 2 diabetes mellitus management: a review of pharmacokinetic and clinical trial data. Drugs R D 7. 2006;(6):331-7. https://doi.org/10.2165/00126839-20060 7060-00002.

12. Tan Z, Xu Z, Gui Q, Wu W, Yang Y. Gliquidone versus metformin: differential effects on aorta in streptozotocin induced diabetic rats. Chin Med J (Engl). 2014;127(7):1298-303.

13. Ke JT, Li M, Xu SQ, Zhang WJ, Jiang YW, Cheng LY, et al. Gliquidone decreases urinary protein by promoting tubular reabsorption in diabetic Goto-Kakizaki rats. J Endocrinol. 2014;220(2):129-41. https://doi.org/10. 1530/JOE-13-0199. 
14. Winnik S, Auwerx J, Sinclair DA, Matter CM. Protective effects of sirtuins in cardiovascular diseases: from bench to bedside. Eur Heart J. 2015;36(48):3404-12. https://doi.org/10.1093/eurheartj/ehv290.

15. Jiang T, Gu J, Chen W, Chang Q. Resveratrol inhibits high-glucose-induced inflammatory "metabolic memory" in human retinal vascular endothelial cells through SIRT1-dependent signaling. Can J Physiol Pharmacol. 2019;97(12):1141-51. https://doi.org/10.1139/cjpp-2019-0201.

16. Ji Q, Han J, Wang L, Liu J, Dong Y, Zhu K, Shi L. MicroRNA-34a promotes apoptosis of retinal vascular endothelial cells by targeting SIRT1 in rats with diabetic retinopathy. Cell Cycle. 2020;19(21):2886-6. https://doi.org/ 10.1080/15384101.2020.1827509.

17. Yang JJ, Tao H, Liu LP, Hu W, Deng ZY, Li J. miR-200a controls hepatic stellate cell activation and fibrosis via SIRT1/Notch1 signal pathway. Inflamm Res. 2017;66(4):341-52. https://doi.org/10.1007/s00011-016-1020-4.

18. Cao YW, Li WQ, Wan GX, Li YX, Du XM, Li YC, et al. Correlation and prognostic value of SIRT1 and Notch1 signaling in breast cancer. J Exp Clin Cancer Res. 2014;33:97. https://doi.org/10.1186/s13046-014-0097-2.

19. Xie M, Liu M, He CS. SIRT1 regulates endothelial Notch signaling in lung cancer. PLoS One. 2012;7(9):e45331. https://doi.org/10.1371/journal.pone. 0045331.

20. Xie H, Sun J, Chen Y, Zong M, Li S, Wang Y. EGCG Attenuates Uric AcidInduced Inflammatory and Oxidative Stress Responses by Medicating the NOTCH Pathway. Oxid Med Cell Longev. 2015;2015:214836. https://doi. org/10.1155/2015/214836.

21. Bray SJ. Notch signalling: a simple pathway becomes complex. Nat Rev Mol Cell Biol. 2006;7(9):678-9. https://doi.org/10.1038/nrm2009.

22. Dou GR, Wang L, Wang YS, Han H. Notch signaling in ocular vasculature development and diseases. Mol Med. 2012;18:47-55. https://doi.org/10. 2119/molmed.2011.00256.

23. Miloudi K, Oubaha M, Menard C, Dejda A, Guber V, Cagnone G, Wilson AM, Tetreault N, Mawambo G, Binet F, Chidiac R, Delisle C, Buscarlet M, Cerani A, Crespo-Garcia S, Bentley K, Rezende F, Joyal JS, Mallette FA, Gratton JP, Larrivee B, Sapieha P. NOTCH1 signaling induces pathological vascular permeability in diabetic retinopathy. Proc Natl Acad Sci U S A. 2019:1 16(10):4538-47. https://doi.org/10.1073/pnas.1814711116.

24. Bai X, He T, Liu Y, Zhang J, Li X, Shi J, et al. Acetylation-Dependent Regulation of Notch Signaling in Macrophages by SIRT1 Affects Sepsis Development. Front Immunol. 2018;9:762. https://doi.org/10.3389/fimmu.2018. 00762.

25. Tian H, Yang J, Xie Z, Liu J. Gliquidone Alleviates Diabetic Nephropathy by Inhibiting Notch/Snail Signaling Pathway. Cell Physiol Biochem. 2018;51(5):2085-97. https://doi.org/10.1159/000495827.

26. Colwell DR, Higgins JA, Denyer GS. Incorporation of 2-deoxy-D-glucose into glycogen. Implications for measurement of tissue-specific glucose uptake and utilisation. Int J Biochem Cell Biol. 1996;28(1):115-21. doi:1357272595001 107 [pii] https://doi.org/10.1016/1357-2725(95) 00110-7.

27. Rodriguez ML, Perez S, Mena-Molla S, Desco MC, Ortega AL. Oxidative Stress and Microvascular Alterations in Diabetic Retinopathy: Future Therapies. Oxid Med Cell Longev. 2019;2019:4940825. https://doi.org/10. 1155/2019/4940825.

28. Forrester JV, Kuffova L, Delibegovic M. The Role of Inflammation in Diabetic Retinopathy. Front Immunol. 2020;1 1:583687. https://doi.org/10. 3389/fimmu.2020.583687.

29. Zhu DD, Wang YZ, Zou C, She XP, Zheng Z. The role of uric acid in the pathogenesis of diabetic retinopathy based on Notch pathway. Biochem Biophys Res Commun. 2018;503(2):921-9. https://doi.org/10.1016/j.bbrc. 2018.06.097.

30. Kida Y, Zullo JA, Goligorsky MS. Endothelial sirtuin 1 inactivation enhances capillary rarefaction and fibrosis following kidney injury through Notch activation. Biochem Biophys Res Commun. 2016;478(3):1074-9. https:// doi.org/10.1016/j.bbrc.2016.08.066.

31. Simo R, Sundstrom JM, Antonetti DA. Ocular Anti-VEGF therapy for diabetic retinopathy: the role of VEGF in the pathogenesis of diabetic retinopathy. Diabetes Care. 2014;37(4):893-9. https://doi.org/10.2337/ dc13-2002.37/4/893.

32. Aiello LP, Bursell SE, Clermont A, Duh E, Ishii H, Takagi C, et al. Vascular endothelial growth factor-induced retinal permeability is mediated by protein kinase $\mathrm{C}$ in vivo and suppressed by an orally effective betaisoform-selective inhibitor. Diabetes. 1997;46(9):1473-80. https://doi.org/ 10.2337/diab.46.9.1473.
33. Frank RN (2008) Diabetic Retinopathy. New England Journal of Medicine

34. Garcia C, Aranda J, Arnold E, Thebault S, Macotela Y, Lopez-Casillas F, et al. Vasoinhibins prevent retinal vasopermeability associated with diabetic retinopathy in rats via protein phosphatase $2 \mathrm{~A}$-dependent eNOS inactivation. J Clin Invest. 2008;118(6):2291-300. https://doi.org/10.1172/JCl34 508.

35. Zhang J, Zhang $X$, Zou Y, Han F. CPSF1 mediates retinal vascular dysfunction in diabetes mellitus via the MAPK/ERK pathway. Arch Physiol Biochem. 2020:1-8. https://doi.org/10.1080/13813455.2020.1722704.

36. Long L, Li Y, Y Y S, Li X, Hu Y, Long T, et al. Scutellarin Prevents Angiogenesis in Diabetic Retinopathy by Downregulating VEGF/ERK/FAK/Src Pathway Signaling. J Diabetes Res. 2019;2019:4875421. https://doi.org/10. 1155/2019/4875421.

37. Antonetti DA, Klein R, Gardner TW. Diabetic retinopathy. N Engl J Med. 2012;366(13):1227-39. https://doi.org/10.1056/NEJMra1005073.

38. Zhu L, Wang W, Xie TH, Zou J, Nie X, Wang X, et al. TGR5 receptor activation attenuates diabetic retinopathy through suppression of RhoA/ROCK signaling. FASEB J. 2020;34(3):4189-203. https://doi.org/10.1096/f.20190 2496RR.

39. Li S, Yang $H$, Chen $X$. Protective effects of sulforaphane on diabetic retinopathy: activation of the Nrf2 pathway and inhibition of NLRP3 inflammasome formation. Exp Anim. 2019;68(2):221-31. https://doi.org/ 10.1538/expanim.18-0146.

40. Wong TY, Cheung CM, Larsen M, Sharma S, Simo R. Diabetic retinopathy. Nat Rev Dis Primers. 2016;2:16012. https://doi.org/10.1038/nrdp.2016.12.

41. Song $Y$, Huang $L$, Yu J. Effects of blueberry anthocyanins on retinal oxidative stress and inflammation in diabetes through $\mathrm{Nrf2/HO}-1$ signaling. J Neuroimmunol. 2016;301:1-6. https://doi.org/10.1016/j.jneuroim.2016.11. 001.

42. Chen Y, Meng J, Li H, Wei H, Bi F, Liu S, et al. Resveratrol exhibits an effect on attenuating retina inflammatory condition and damage of diabetic retinopathy via PON1. Exp Eye Res. 2019;181:356-66. https://doi.org/10. 1016/j.exer.2018.11.023.

43. Jiang T, Chang Q, Cai J, Fan J, Zhang X, Xu G. Protective Effects of Melatonin on Retinal Inflammation and Oxidative Stress in Experimental Diabetic Retinopathy. Oxid Med Cell Longev. 2016;2016:3528274. https:// doi.org/10.1155/2016/3528274.

44. An L, Li Z, Shi L, Wang L, Wang Y, Jin L, et al. Inflammation-Targeted Celastrol Nanodrug Attenuates Collagen-Induced Arthritis through NF-kappaB and Notch1 Pathways. Nano Lett. 2020;20(10):7728-36. https://doi.org/ 10.1021/acs.nanolett.0c03279.

45. Frick A, Khare V, Jimenez K, Dammann K, Lang M, Krnjic A, Gmainer C, Baumgartner M, Mesteri I, Gasche C. A Novel PAK1-Notch1 Axis Regulates Crypt Homeostasis in Intestinal Inflammation. Cell Mol Gastroenterol Hepatol. 2021;11(3):892-907 e891. https://doi.org/10.1016/j.jcmgh.2020. 11.001.

46. Fang Z, Wu G, Zhang D, Wang K, Deng X, Liu M, et al. Genistein Protects Against Burn-Induced Myocardial Injury via Notch1-Mediated Suppression of Oxidative/Nitrative Stress. Shock. 2020;54(3):337-46. https://doi. org/10.1097/SHK.0000000000001464.

47. Luo J, Li L, Hu D, Zhang X. LINC00612/miR-31-5p/Notch1 Axis Regulates Apoptosis, Inflammation, and Oxidative Stress in Human Pulmonary Microvascular Endothelial Cells Induced by Cigarette Smoke Extract. Int J Chron Obstruct Pulmon Dis. 2020;15:2049-60. https://doi.org/10.2147/ COPD.S255696.

48. Lazzara F, Fidilio A, Platania CBM, Giurdanella G, Salomone S, Leggio GM, Tarallo V, Cicatiello V, De Falco S, Eandi CM, Drago F, Bucolo C. Aflibercept regulates retinal inflammation elicited by high glucose via the PIGF/ ERK pathway. Biochem Pharmacol. 2019;168:341-51. https://doi.org/10. 1016/j.bcp.2019.07.021.

49. Giurdanella G, Anfuso CD, Olivieri M, Lupo G, Caporarello N, Eandi CM, Drago F, Bucolo C, Salomone S. Aflibercept, bevacizumab and ranibizumab prevent glucose-induced damage in human retinal pericytes in vitro, through a PLA2/COX-2/NEGF-A pathway. Biochem Pharmacol. 2015;96(3):278-87. https://doi.org/10.1016/j.bcp.2015.05.017.

50. Wang $Y$, Tao J, Jiang $M$, Yao $Y$. Apocynin ameliorates diabetic retinopathy in rats: Involvement of TLR4/NF-kappaB signaling pathway. Int Immunopharmacol. 2019;73:49-56. https://doi.org/10.1016/j.intimp.2019.04.062.

51. He Y, Dan Y, Gao X, Huang L, Lv H, Chen J. DNMT1-mediated IncRNA MEG3 methylation accelerates endothelial-mesenchymal transition in diabetic retinopathy through the PI3K/Akt/mTOR signaling pathway. Am 
J Physiol Endocrinol Metab. 2021;320(3):E598-608. https://doi.org/10. 1152/ajpendo.00089.2020.

52. Platania CBM, Maisto R, Trotta MC, D'Amico M, Rossi S, Gesualdo C, et al. Retinal and circulating miRNA expression patterns in diabetic retinopathy: An in silico and in vivo approach. Br J Pharmacol. 2019;176(13):217994. https://doi.org/10.1111/bph.14665.

53. Lazzara F, Trotta MC, Platania CBM, D'Amico M, Petrillo F, Galdiero M, et al. Stabilization of HIF-1alpha in Human Retinal Endothelial Cells Modulates Expression of miRNAs and Proangiogenic Growth Factors. Front Pharmacol. 2020;11:1063. https://doi.org/10.3389/fphar.2020.01063.

54. Biswas S, Sarabusky M, Chakrabarti S. Diabetic Retinopathy, IncRNAs, and Inflammation: A Dynamic, Interconnected Network. J Clin Med. 2019;8(7). https://doi.org/10.3390/jcm8071033.

55. Chen Y, He Y, Zhou H. The potential role of IncRNAs in diabetes and diabetic microvascular complications. Endocr J. 2020;67(7):659-68. https:// doi.org/10.1507/endocri.EJ19-0574.

\section{Publisher's Note}

Springer Nature remains neutral with regard to jurisdictional claims in published maps and institutional affiliations.

- fast, convenient online submission

- thorough peer review by experienced researchers in your field

- rapid publication on acceptance

- support for research data, including large and complex data types

- gold Open Access which fosters wider collaboration and increased citations

- maximum visibility for your research: over $100 \mathrm{M}$ website views per year

At BMC, research is always in progress.

Learn more biomedcentral.com/submissions 